\title{
Análise de Desempenho de Tráfego Multimídia em redes PLC utilizando recomendações do ITU-T
}

\author{
Amaro Krob ${ }^{1}$, Leandro Cassol ${ }^{1}$, Andrea Collin ${ }^{1}$ \\ ${ }^{1}$ Universidade La Salle \\ Av. Victor Barreto, 2288 - Canoas - RS - Brasil \\ amarokrob@gmail.com.br, \{cassol, andrea\}@unilasalle.edu.br
}

\begin{abstract}
This work presents a performance analysis of the PLC technology when submitted to multimedia traffics in real time. Uses the ITU-T (International Telecommunication Union) $G$ and $Y$ series recommendations as reference models to compose the environment and metrics of this experiment. The environment chosen for conducting the analysis is an indoor residential network, the results obtained are in accordance with the ITU-T reference model E presented through the scalar factor $R$ and also in the MOS (Mean Opinion Score) standard.

Resumo. Este trabalho apresenta uma análise de desempenho da tecnologia PLC quando submetida a tráfegos multimidia em tempo real. Utiliza as recomendações do ITU-T (International Telecommunication Union) série G e $Y$ como modelos de referência para compor o ambiente e as métricas deste experimento. O ambiente escolhido para realização da análise é uma rede de abrangência residencial indoor, os resultados obtidos estão conforme o modelo E de referência do ITU-T apresentados através do fator escalar $R e$ também no critério MOS (Mean Opinion Score).
\end{abstract}

\section{Introdução}

O PLC (Power Line Communication) é uma tecnologia de comunicação que utiliza a rede de energia elétrica para o envio dos dados. A principal vantagem desta tecnologia é a utilização da infraestrutura já existente, não necessitando a construção de novos meios físicos para o transporte de informações [Vieira e Alves 2015]. O elevado custo para implantação de novas redes destinadas a comunicações multimídias em instalações residenciais serviu de motivação para o desenvolvimento deste experimento.

A tecnologia PLC utiliza a rede de energia elétrica, em baixa ou média tensão, operando dentro da faixa de 1,705 até $80 \mathrm{MHz}$ para prover serviços de comunicação de dados, voz e vídeo. Na rede PLC é possível encontrar aplicações como conexão à internet, IoT (Internet of Things), Smart Grid, segurança IP, voz sobre IP, entre outros.

O PLC atua na camada 2 do modelo TCP/IP e pode ser agregado a uma rede IP já existente, trabalhando em conjunto com outras tecnologias [Camargo 2010]. Devido às características de funcionamento das redes IPs (Best Effort), problemas como o atraso, jitter, perda e ordenação de pacotes podem influenciar a qualidade das transmissões. Aplicações multimídia como a voz sobre IP (VoIP) exigem que a informação seja transportada em tempo real para não comprometer a experiência do usuário com este tipo de serviço. 
Com o crescimento das aplicações de IoT, os requisitos para comunicações estão cada vez mais diversificados. Alguns aplicativos IoT, como aplicativos de vídeo 8k / 4k para entretenimento ou segurança precisam transmitir dados em velocidades altas. Outros aplicativos, como IoT para cidades inteligentes, gerenciamento de energia, controle de edifícios, controle de iluminação, necessitam de comunicação de longa distância em vez de velocidade alta. Os diferentes modos especificados no padrão [IEEE P1901.3] abordam essa diversidade de requisitos, sendo essa norma complementar aos padrões [IEEE 1901], [IEEE P1901.1] e [IEEE 1901.2].

Em dezembro de 2017 com a criação do grupo de trabalho do padrão [IEEE P1901.3] iniciou-se a especificação da IoTPLC, baseada em Wavelet OFDM (Wavelet Orthogonal Frequency Division Multiplexing). Esta norma define modos para operações em diferentes canais (bandas de frequência) com diferentes valores de espaçamento da portadora. O IoTPLC pode ser usado para comunicações com fio em qualquer tipo de meio físico, como linhas de energia elétrica e cabos coaxiais. Esse padrão usa frequências de transmissão abaixo de $100 \mathrm{MHz}$ e aborda as questões de segurança necessárias para garantir a privacidade das comunicações entre usuários, permitindo o uso do IoTPLC para serviços sensíveis à segurança. Um canal de comunicação definido nesta norma interopera com o padrão [IEEE 1901].

Este artigo apresenta uma análise do desempenho de transmissões multimídia através da tecnologia PLC, com o objetivo de validar a rede de energia elétrica residencial como um meio físico apto a estabelecer comunicações em tempo real.

Para validar o desempenho da rede PLC, foi realizado um experimento com a transmissão de dados caracterizados como fluxos de voz sobre IP, utilizando como referência de análise o modelo E do [ITU-T G.107 2015] e as métricas de atraso fim-afim, jitter e perdas.

Este trabalho está organizado da seguinte maneira: a seção 2 descreve a tecnologia PLC e as suas principais características, a seção 3 apresenta os requisitos de QoS (Quality Of Service) das aplicações multimídia, bem como as recomendações do ITU-T utilizadas nesta pesquisa. A descrição completa do modelo E do ITU-T é realizada na seção 4. A seção 5 especifica a metodologia utilizada nos experimentos, a seção 6 aborda a aplicação das recomendações ITU-T nesta análise. Na seção 7 são apresentados os resultados encontrados e por fim, a seção 8 aborda as considerações finais desta pesquisa.

\section{A rede $\mathrm{PLC}$}

Nesta seção será apresentada a tecnologia PLC, bem como suas principais características e avanços nas aplicações de comunicação de dados através da rede de energia elétrica em ambiente residencial indoor.

A tecnologia PLC é uma solução que vem apresentando uma crescente evolução no que tange ao modelo OFDM. Conforme [Figueiredo 2015], Judy Gorman managing director IEEE Standards Association, membro do grupo de trabalho do [IEEE P.1901], responsável pelo desenvolvimento do padrão global para comunicações através da rede elétrica: "A tecnologia PLC tem se apresentado como mais uma opção tecnológica alternativa para as redes convencionais como o Ethernet ou Wireless. Oferecendo considerável vantagem por passar entre as paredes e outras estruturas que obstruem o sinal de radiofrequência”, disse. 
Como a rede elétrica está presente em praticamente todos os lugares, não há necessidade de obras para instalação de cabos adicionais para prover serviços de dados em uma instalação já existente [Zattar 2011].

\section{Qualidade de serviço (QoS) e recomendações ITU-T}

A transmissão de voz sobre redes de pacotes traz novas formas de degradação da qualidade da comunicação. Existem diversas definições para controlar os parâmetros de desempenho visando obter QoS para aplicações em tempo real, dentre elas destacam-se as recomendações do ITU-T.

A recomendação [ITU-T G.1020 2006] define a qualidade das chamadas VoIP utilizando redes IP, bem como os parâmetros de desempenho para garantir a qualidade das transmissões. O padrão do ITU-T concentra-se nas deficiências resultantes das variações no atraso e perdas de pacotes, pois é o que gera impacto direto nas aplicações em tempo real. Essa recomendação sugere o uso de mecanismos de compensação, tais como buffers para amenização do jitter e ocultação das perdas para reduzir o impacto sobre a qualidade da voz.

A Figura 1 apresenta uma visão geral da análise do fluxo de dados na rede de pacotes, proporcionando um completo entendimento do escopo, bem como as interações entre as recomendações que compõem a análise de desempenho realizada neste trabalho.

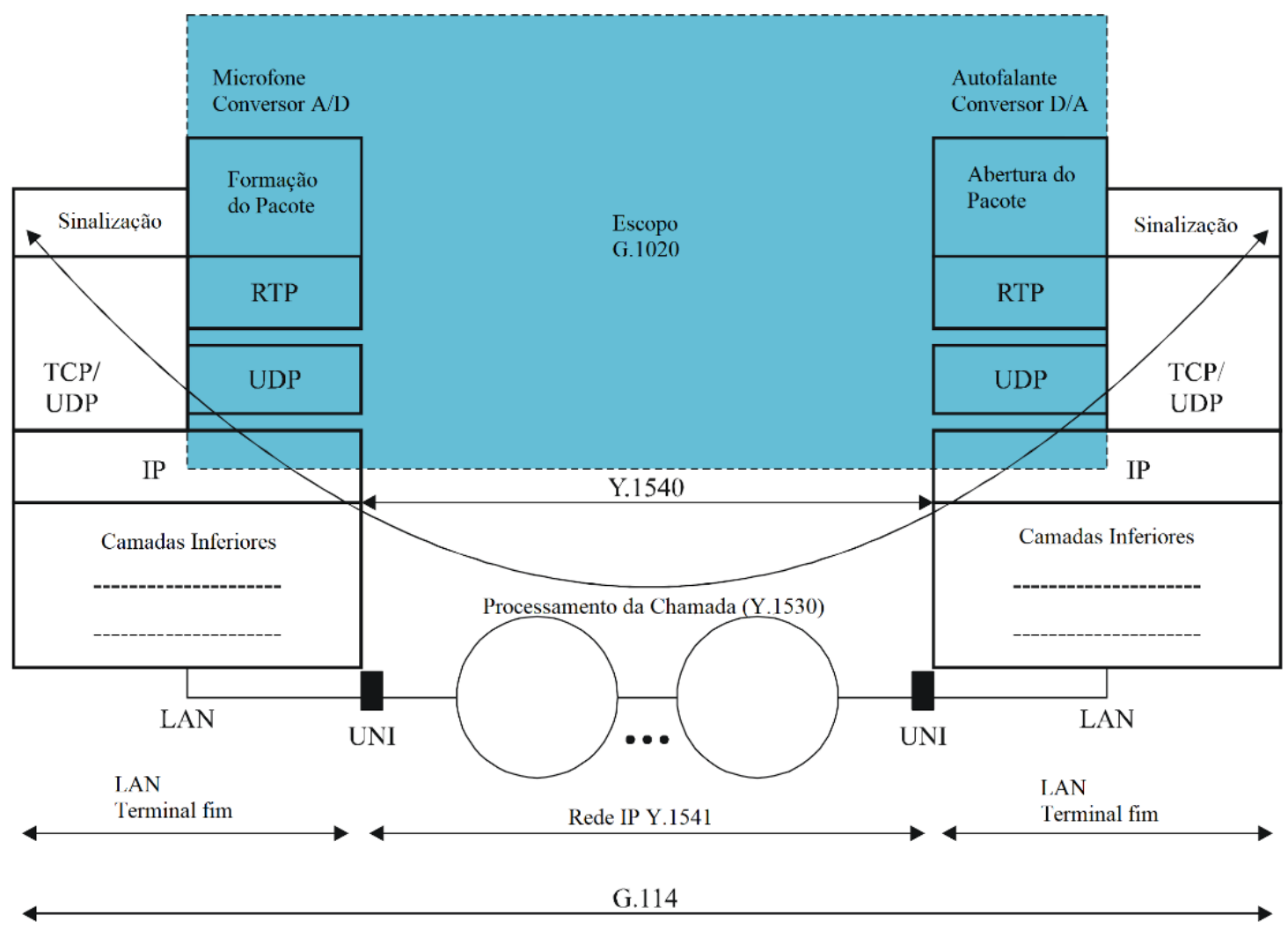

Figura 1: Escopo da recomendação [ITU-T G.1020 2006] em relação a outros padrões.

Na Figura 1 observa-se que em conjunto com a recomendação da série G do ITUT, encontra-se a recomendação [ITU-T Y.1540 2016] que define parâmetros que podem ser usados para avaliar o desempenho de velocidade, precisão, confiabilidade e 
disponibilidade de transferência de pacotes em redes de comunicação de dados com o protocolo IP.

$\mathrm{Na}$ parte relacionada a qualidade das comunicações fim-a-fim, adota-se os parâmetros definidos na recomendação [ITU-T Y.1541 2016] que possui exemplos detalhados sobre a utilização do modelo E para avaliar o desempenho de transmissão de dados que envolvem problemas como atraso, jitter e perdas.

A metodologia utilizada neste trabalho está complementarmente referenciada na recomendação [ITU-T G.1050 2016] que aborda uma transmissão multimídia sobre protocolo IP, utilizando-a para avaliar o desempenho com foco no atraso do pacote, na variação do atraso e na perda. Esta análise considera que o jitter máximo deve ser menor que $50 \mathrm{~ms}$ e as perdas limitadas a $3 \%$ (recomendável $<0,5 \%$ ). Para uma experiência de boa qualidade em VoIP, a recomendação [ITU-T G.107 2015] define que o valor de referência para o atraso fim-a-fim deve ser menor que $150 \mathrm{~ms}$.

\section{O modelo E do ITU-T}

Para analisar o desempenho de chamadas VoIP, bem como determinar a satisfação do usuário durante uma ligação, existem duas categorias de modelos: os subjetivos e os objetivos, ambos são padronizados através das recomendações do ITU-T.

O modelo subjetivo é oriundo da avaliação pessoal da experiência do usuário e tem como exemplo o padrão [ITU-T P.800 1996] e [ITU-T P.830 1996]. O modelo objetivo, por outro lado, visa analisar a influência de parâmetros como o atraso fim-a-fim, jitter e perdas na qualidade da chamada, como é o caso dos padrões [ITU-T G.107 2015] e [ITU-T G.108 2008].

O modelo E encontra-se na categoria dos modelos objetivos e foi padronizado na recomendação [ITU-T G.107 2015], conforme apresenta a Figura 2. Esse modelo foi aplicado neste trabalho para obtenção dos resultados em conjunto com a recomendação [ITU-T G.108 2008].

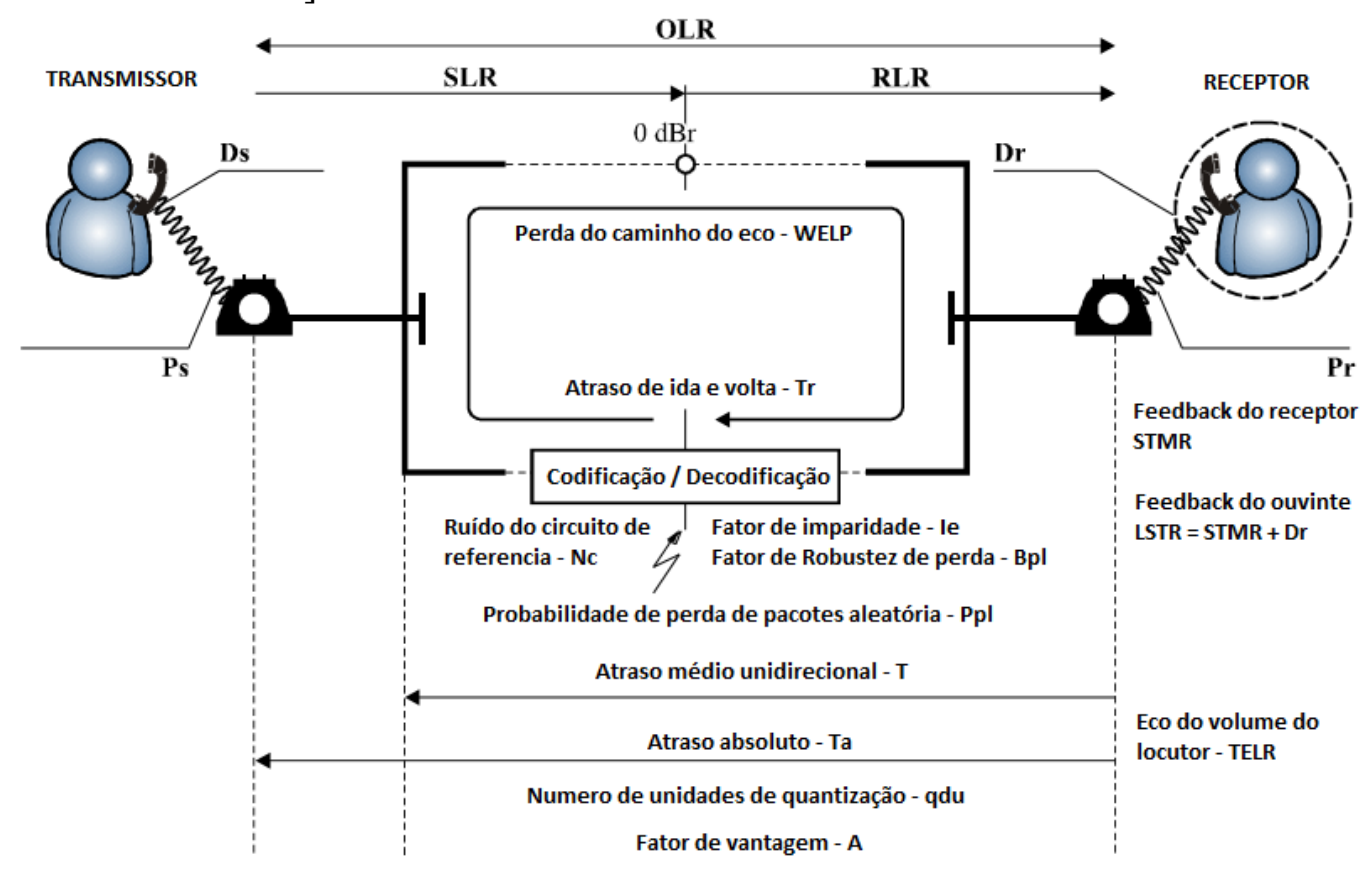

Figura 2: Padrão do Modelo E [ITU-T G.107 2015]. 
A tabela 1 a seguir apresenta os valores recomendados pelo padrão [ITU-T G.107 2015], que foram adotados no cálculo do fator R das chamadas VoIP analisadas.

Tabela 1: Valores padrão para os parâmetros do Modelo E [ITU-T G.107 2015].

\begin{tabular}{|l|c|c|c|c|}
\hline Parâmetros & Sigla & Und & Valor Adotado & Variação Permitida \\
\hline Volume enviado & $\mathrm{SLR}$ & $\mathrm{dB}$ & +8 & $0 \ldots+18$ \\
\hline Volume recebido & $\mathrm{RLR}$ & $\mathrm{dB}$ & +2 & $-5 \ldots+14$ \\
\hline Feedback do receptor & $\mathrm{STMR}$ & $\mathrm{dB}$ & 15 & $10 \ldots 20$ \\
\hline Feedback do ouvinte & $\mathrm{LSTR}$ & $\mathrm{dB}$ & 18 & $13 \ldots 23$ \\
\hline Valor D enviado & $\mathrm{Ds}$ & - & 3 & $-3 \ldots+3$ \\
\hline Valor D recebido & $\mathrm{Dr}$ & - & 3 & $-3 \ldots+3$ \\
\hline Eco do volume do locutor & $\mathrm{TELR}$ & $\mathrm{dB}$ & 65 & $5 \ldots 65$ \\
\hline Perda do caminho do eco & $\mathrm{WEPL}$ & $\mathrm{dB}$ & 110 & $5 \ldots 110$ \\
\hline Atraso médio unidirecional & $\mathrm{T}$ & $\mathrm{ms}$ & $\mathrm{Calculado}$ & $0 \ldots 500$ \\
\hline Atraso de ida e volta & $\mathrm{Tr}$ & $\mathrm{ms}$ & $\mathrm{Medido}$ & $0 \ldots 1000$ \\
\hline Atraso absoluto & $\mathrm{Ta}$ & $\mathrm{ms}$ & $\mathrm{TP}+\mathrm{T}+\mathrm{TB}$ & $0 \ldots 500$ \\
\hline Número de unidades de quantização & $\mathrm{qdu}$ & - & 1 & $1 \ldots 14$ \\
\hline Fator de imparidade de equipamentos & $\mathrm{le}$ & - & 0 & $0 \ldots 40$ \\
\hline Fator de robustez de perda de pacotes & $\mathrm{Bpl}$ & - & 4.8 & $4.3 \ldots 40$ \\
\hline Probabilidade de perda de pacote aleatória & $\mathrm{Ppl}$ & $\%$ & 0 & $0 \ldots 20$ \\
\hline Ruído de circuito de referência & $\mathrm{Nc}$ & $\mathrm{dBm0p}$ & -70 & $-80 \ldots-40$ \\
\hline Ruído no lado da recepção & $\mathrm{Pr}$ & $\mathrm{dBmp}$ & 35 & - \\
\hline Ruído no lado do envio & $\mathrm{Ps}$ & $\mathrm{dB}(\mathrm{A})$ & 35 & $35 \ldots 85$ \\
\hline
\end{tabular}

Através do modelo E é obtido o fator escalar R, que varia de 0 (péssimo) a 100 (excelente) indicando a satisfação do usuário.

O modelo E implementa um mecanismo baseado na soma de termos que representam distorções na qualidade da voz, tais como atrasos de transmissão, eco, distorções introduzidas pelos equipamentos, conforme a equação (1.1) a seguir.

$$
R=R o-I s-I d-I e+A
$$

Valores conforme [ITU-T G.107 2015], [Braga 2010]:

- $\quad \mathbf{R o}=$ 94,77: representa os efeitos da relação SNR (sinal ruído), composto por fontes de ruído, tais como ruídos de circuitos de transmissão e ruído ambiente;

- Is = 1,41: representa as perdas simultâneas ao sinal de voz digitalizado, composto por perdas devido ao excesso de volume, perdas ocasionadas durante o caminho que a voz do locutor percorre até seu microfone e perdas causadas pela distorção de quantização, sofridas durante o processo de digitalização e codificação da voz;

- $\mathbf{I d}=\mathbf{T a}=$ TPacket $+\mathbf{T}+$ Tbuffer: são as perdas associadas ao atraso fim a fim, compreendendo a soma de perdas por características próprias dos equipamentos transmissor e receptor;

- $\mathbf{I e}=\mathbf{0}$ : representa as perdas associadas a tecnologia utilizada, sendo dependente do tipo de Codec, no caso de VoIP G.711 é igual a zero; 
- $\mathbf{A}=\mathbf{0}$ : corresponde ao fator de vantagem ou fator de expectativa, definindo um grau de tolerância que o usuário espera pelo uso da tecnologia. Por exemplo, se os utilizadores estão cientes de que estão se comunicando utilizando link satélite, serão mais tolerantes com a degradação devido aos longos atrasos.

$\mathrm{Na}$ literatura também são encontrados resultados apresentados no critério MOS, baseado nos modelos subjetivos. A Figura 3 apresenta a relação entre o fator escalar R e o critério MOS, enquanto a equação (1.2) realiza a conversão do fator R para o fator MOS equivalente.

$$
M O S=1+0,035 \cdot R+R \cdot(R-60) \cdot(100-R) \cdot 7 E^{-6}
$$

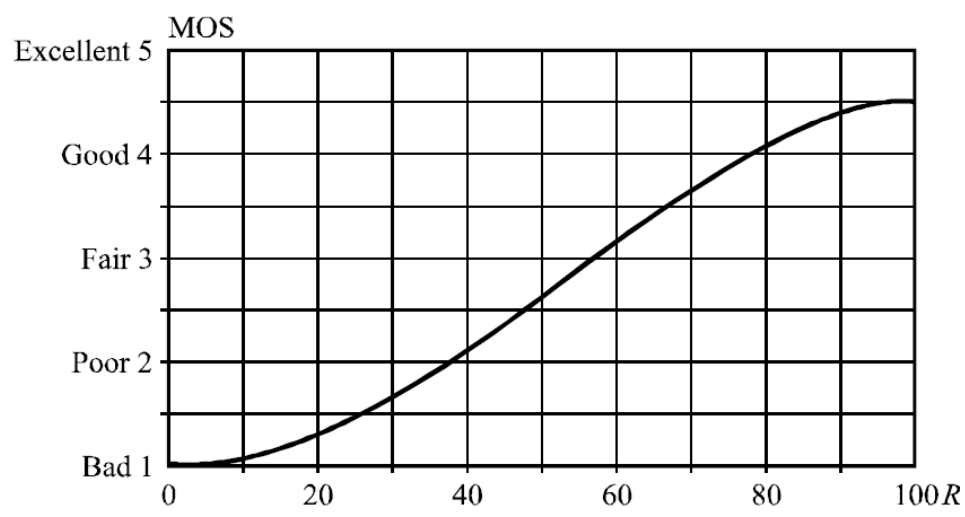

Figura 3: Fator R x Critério MOS [ITU-T G.107 2015]

Detalhes sobre cálculos dos parâmetros do modelo E, bem como as equações apresentadas acima podem ser encontrados em [ITU-T G.107 2015].

\section{Metodologia}

Para realizar a análise de desempenho proposta neste trabalho foi utilizada a ferramenta de geração de dados multimídia [JPerf 2016] e para a captura de pacotes do protocolo IP a ferramenta [Wireshark 2016], além de 2 adaptadores PLC/Ethernet, 2 notebooks equipados com placa de rede gigabit e o software Libre Calc para realização da análise matemática e estatística dos dados obtidos.

Devido a necessidade de sincronismo temporal entre o transmissor e receptor durante a comunicação, foi utilizada a função "Net Time" do sistema operacional Windows para sincronizar os relógios de ambos os notebooks com um servidor externo.

A primeira etapa do experimento foi a configuração da ferramenta [JPerf 2016]. A caracterização dos pacotes foi realizada conforme o padrão G.711 [ITU-T G.711 1999] para uma chamada VoIP. A Tabela 1 apresenta a parametrização dos pacotes IP utilizados nesse experimento.

Tabela 1: Pacote IP gerado por uma chamada VoIP [ITU-T G.711 1999].

\begin{tabular}{|c|c|c|c|}
\hline Cabeçalho IP & Cabeçalho UDP & Cabeçalho RTP & Payload (Dados) \\
\hline 20 bytes & 8 bytes & 12 bytes & 160 bytes \\
\hline
\end{tabular}


O cenário utilizado para os testes é apresentado na Figura 4, sendo composto por dois computadores equipados com placas de rede gigabit Ethernet instalados em diferentes cômodos de uma residência, conectados através da rede PLC. As transmissões de dados foram criadas para conjuntos de chamadas de trafego concorrente, variando de 1 a 50 chamadas VoIP crescente em múltiplos de 5, visando gerar, medir e saturar a comunicação para analisar as métricas de atraso fim-a-fim, jitter e perdas de pacotes.

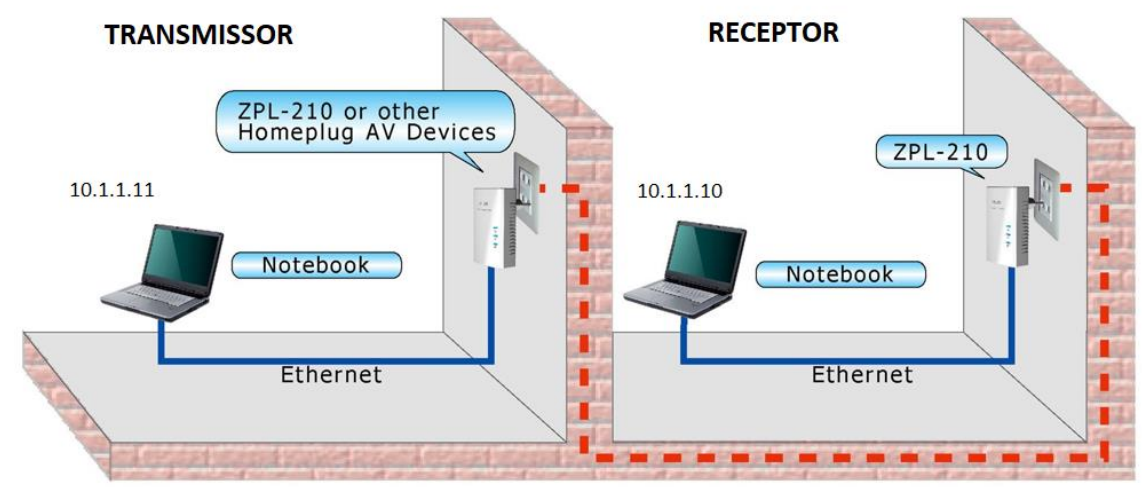

Figura 4: Cenário utilizado para conexão da rede PLC [Zinwell 2016]

A ferramenta [Wireshark 2016] foi utilizada durante as transmissões para capturar os pacotes enviados entre os equipamentos. Essa etapa teve como objetivo armazenar os fluxos gerados para posterior análise, que será abordada a seguir.

\subsection{Coleta de dados}

Ao todo foram capturados 415.000 pacotes de dados em 11 diferentes tipos de transmissões. Esses dados capturados pelo [Wireshark 2016] foram exportados no formato .CSV e importados no Libre Calc, sendo organizados de forma resumida conforme apresentado na Tabela 2.

Tabela 2: Fluxos capturados durante a transmissão [autoria própria].

\begin{tabular}{c|c|c|c|c|c|c|}
\hline \multicolumn{2}{|c|}{ TRANSMISSOR (TX) } & \multicolumn{2}{c|}{ RECEPTOR (RX) } & \multicolumn{3}{c|}{ VALORES CALCULADOS } \\
\hline Pacote & Tempo (s) & Pacote & Tempo (s) & Delay (ms) & Jitter (ms) & |Jitter| \\
\hline 23 & 15,301497 & 23 & 15,305750 & 4,253 & 0 & 0 \\
\hline 24 & 15,329077 & 24 & 15,333327 & 4,250 & $-0,003$ & 0,003 \\
\hline 53 & 15,406925 & 53 & 15,411050 & 4,125 & $-0,125$ & 0,125 \\
\hline 54 & 15,406943 & 54 & 15,411804 & 4,861 & 0,736 & 0,736 \\
\hline 55 & 15,406961 & 55 & 15,411804 & 4,843 & $-0,018$ & 0,018 \\
\hline 56 & 15,406978 & 56 & 15,411805 & 4,827 & $-0,016$ & 0,016 \\
\hline 57 & 15,406999 & 57 & 15,411805 & 4,806 & $-0,021$ & 0,021 \\
\hline
\end{tabular}

Para realizar a análise de desempenho através das recomendações do ITU-T com base no modelo $\mathrm{E}$, foram desenvolvidos os cálculos que serão apresentados no decorrer deste capítulo, referenciados pelos parâmetros do padrão [ITU-T G.107 2015] e demais recomendações citadas anteriormente. 


\subsection{Cálculo do atraso}

Segundo a [RFC 3393 2002], o atraso da rede é a diferença de tempo em que o pacote é recebido no destino em relação ao momento em que o pacote foi enviado pela origem. Este cálculo é apresentado na equação (1.3) e seu resultado pode variar em função da carga da rede.

$$
\operatorname{Atraso}_{(m s)}=[\operatorname{Tempo}(R x)-\operatorname{Tempo}(T x)] \cdot 1000
$$

A amostra dos atrasos apresentada na Tabela 3, foi capturada durante a transmissão de 10 fluxos UDP concorrentes, onde ao todo foram transmitidos 1902 pacotes com tamanho de 202 bytes cada. Os dados capturados foram utilizados na equação 1.3 para calcular o atraso do canal de comunicação PLC.

Com base nos valores calculados, foi realizada a análise de probabilidade estatística para obter uma distribuição dos atrasos em classes de intervalos $\mathrm{Xi}$, conforme apresentado na Tabela 3. O intervalo de maior frequência do atraso no canal PLC encontra-se em $\mathrm{Xi}=5$.

Tabela 3: Distribuição do atraso em intervalos de classes [autoria própria].

\begin{tabular}{c|r|r|r|r|r|}
\hline \multicolumn{3}{|c|}{ INTERVALOS } & \multicolumn{3}{|c|}{ FREQUÊNCIAS } \\
\hline $\mathbf{X i}$ & Delay (ms) & Intervalo & Acumulada & Relativa \\
\hline 1 & 3,721 & 4,020 & 149 & 149 & 0,0783 \\
\hline 2 & 4,020 & 4,318 & 141 & 290 & 0,0741 \\
\hline 3 & 4,318 & 4,617 & 77 & 367 & 0,0405 \\
\hline 4 & 4,617 & 4,915 & 178 & 545 & 0,0936 \\
\hline 5 & 4,915 & 5,214 & 637 & 1182 & 0,3349 \\
\hline 6 & 5,214 & 5,513 & 585 & 1767 & 0,3076 \\
\hline 7 & 5,513 & 5,811 & 86 & 1853 & 0,0452 \\
\hline 8 & 5,811 & 6,110 & 40 & 1893 & 0,0210 \\
\hline 9 & 6,110 & 6,408 & 3 & 1896 & 0,0016 \\
\hline 10 & 6,408 & 6,707 & 5 & 1901 & 0,0026 \\
\hline 11 & 6,707 & 7,005 & 0 & 1901 & 0,0000 \\
\hline 12 & 7,005 & 7,304 & 1 & 1902 & 0,0005 \\
\hline
\end{tabular}

Na tabela 4 é apresentado o desvio padrão, bem como o coeficiente de variação da média e também da mediana, permitindo validar a dispersão da amostra e compará-la com padrão de dispersão $<15 \%$, que é considerado homogêneo.

Tabela 4: Análise estatística do atraso [autoria própria].

\begin{tabular}{|c|c|c|c|}
\hline \multicolumn{4}{|c|}{ ANÁLISE ESTATISTICA DO DELAY } \\
\hline Delay maximo (ms) & Dmax & 7,304 & $\mathrm{~ms}$ \\
\hline Delay minimo (ms) & Dmin & 3,721 & $\mathrm{~ms}$ \\
\hline Amplitude de variação (ms) & A & 3,583 & $\mathrm{~ms}$ \\
\hline Quantidade de Classes & 12 & 11,821 & Classes \\
\hline Amplitude de Classes (ms) & h 0,298583333 & 0,303 & $\mathrm{~ms}$ \\
\hline Posição da Mediana & PosMd & 951,5 & Posição \\
\hline Valor da Mediana & Dmd & 5,125 & $\mathrm{~ms}$ \\
\hline Maior Frequência & Dmoda & 5,209 & $\mathrm{~ms}$ \\
\hline Variância & 52 & 0,252 & \\
\hline Desvio padrão & $\mathrm{S}$ & 0,502 & \\
\hline Media & $X$ & 5,001 & $\mathrm{~ms}$ \\
\hline Coeficiente Variação Media & CV (Media) 0,100310501 & 10,03 & $\%$ \\
\hline Coeficiente Variação Mediana & CV (Mediana) $\quad 0,0978776$ & 9,79 & $\%$ \\
\hline
\end{tabular}


A Figura 5 apresenta um gráfico de distribuição normal dos valores de atraso calculados a partir da equação (1.3) para uma transmissão de 10 fluxos simultâneos.

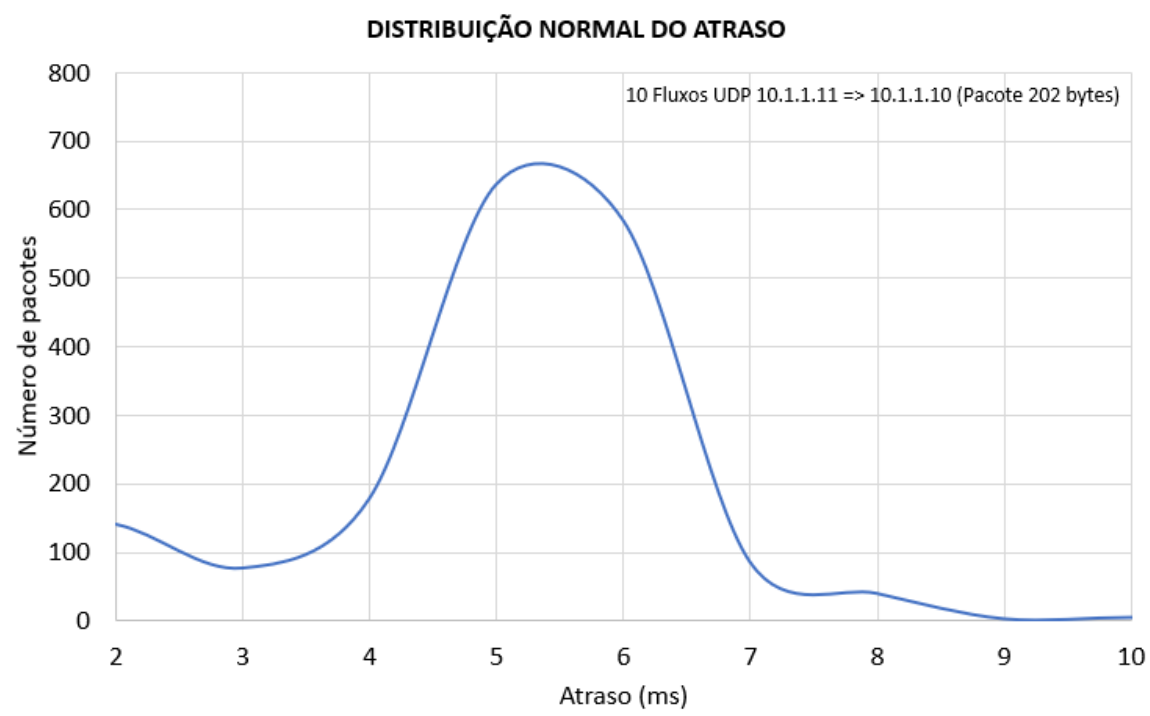

Figura 5: Exemplo de distribuição normal do atraso [autoria própria]

Esta etapa do experimento permitiu a validação da metodologia utilizada na análise das amostras dos fluxos de dados capturados durante as transmissões. Os dados obtidos e analisados serão apresentados na seção de resultados.

\subsection{Cálculo do Jitter}

O cálculo do jitter apresentado na equação (1.4) levou em consideração o padrão estabelecido pela [RFC 3393 2002]. Neste caso, calcula-se a diferença entre o tempo de recebimento de dois pacotes consecutivos e o intervalo de tempo de geração destes mesmos pacotes [Braga 2010], ou seja, é a variação do tempo de recebimento entre dois pacotes a e b.

$$
\operatorname{Jitter}[a, b]_{(m s)}=[\operatorname{Tempo}(R x b-R x a)]-[T e m p o(T x b-T x a)]
$$

Levando em consideração que o jitter é uma taxa de variação que pode oscilar entre valores positivos e negativos, foi utilizado o módulo do mesmo para obter o valor máximo da variação do atraso. A Tabela 5 apresenta a análise do jitter realizada neste trabalho. Após determinar o |Jitter $\mid$ foi obtida a maior amplitude da variação, a Mediana, a Moda e a Média para o intervalo de amostragem do fluxo de dados.

Tabela 5: Análise do Jitter em módulo [autoria própria].

\begin{tabular}{|l|c|c|c|}
\hline \multicolumn{2}{|c|}{ A nálise do Jitter } & Medido & Adotado \\
\hline Jitter Máximo & Max & 3,069 & 3,357 \\
\hline Jitter Mínimo & Min & $-3,357$ & 0,000 \\
\hline Valor da Mediana & Md & & 0,018 \\
\hline Maior Frequência & Moda & & 0,017 \\
\hline Media & $\mathrm{X}$ & & 0,123 \\
\hline
\end{tabular}




\subsection{Cálculo das Perdas de Pacotes}

Para determinar as perdas foi realizada uma comparação da quantidade de pacotes transmitidos com a quantidade de pacotes recebidos, sendo a diferença entre eles o resultado do número de pacotes perdidos durante a transmissão.

A perda de pacotes tem influência direta na qualidade de uma chamada VoIP, pois os pacotes perdidos durante uma transmissão UDP e RTP não podem ser retransmitidos, visto que seriam recebidos fora de ordem e fora do tempo, o que não é admissível para transmissões em tempo real.

\section{Aplicando as recomendações do ITU-T}

A recomendação [ITU-T G.114 2003] prevê limites e valores para o atraso médio de sentido único, independente de outras dificuldades da transmissão. A necessidade de considerar os efeitos combinados de todas as deficiências e qualidades da transmissão a partir do modelo E é apresentada na recomendação [ITU-T G.107 2015] comumente utilizado para classificação de qualidade de chamadas de voz.

Através da recomendação [ITU-T G.108 2008] obtém-se exemplos detalhados sobre a utilização do modelo E na avaliação de desempenho de transmissões em redes de pacotes que envolvem as métricas de atraso, jitter e perdas.

\subsection{Modelo de referência}

O cenário da Figura 6 apresenta o modelo de referência da recomendação [ITU-T Y.1541 2011] utilizado neste trabalho. O mesmo descreve uma ligação entre dois terminais VoIP através de uma rede de pacotes LAN com um atraso total fixo de $100 \mathrm{~ms}$.

Em substituição ao atraso fixo da LAN, assume-se neste experimento o atraso real da rede obtido na medição e calculado na seção 5.2, a partir deste momento denominado TAtrasoLan.

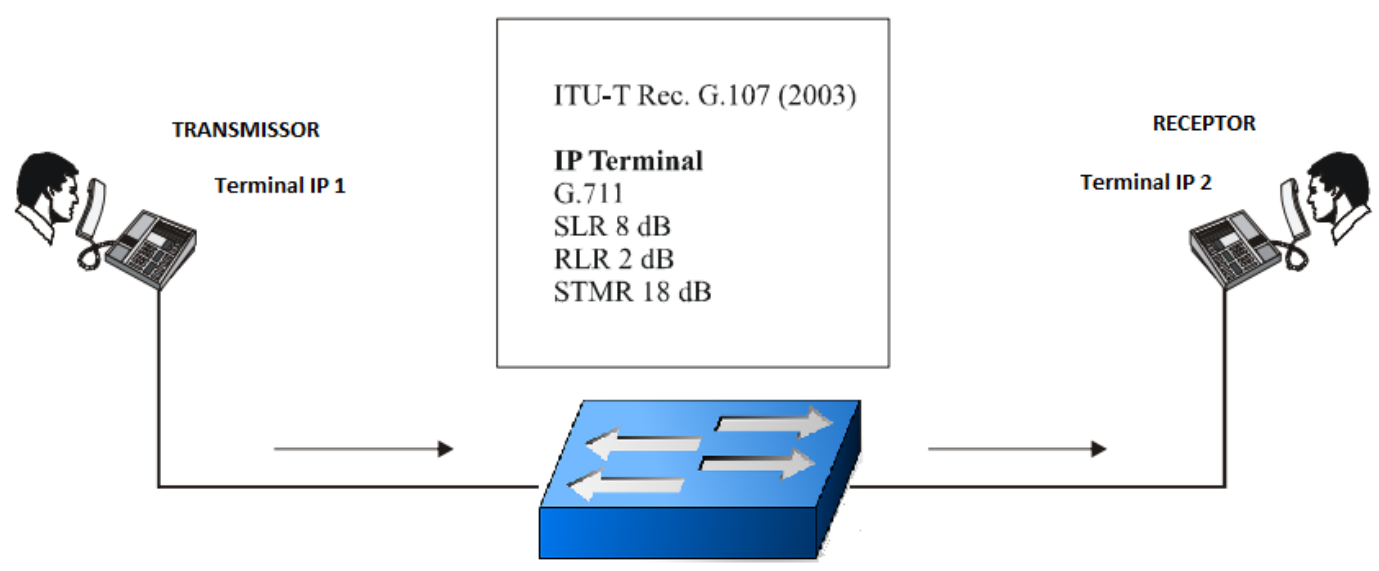

Figura 6: Transmissão com G.711 [ITU-T Y.1541 2011]. 


\subsection{Determinando o Fator $\mathbf{R}$}

As premissas adotadas para determinar o fator R são apresentadas na Tabela 8, bem como a equação (1.5) utilizada para realização do cálculo do parâmetro $\mathrm{T}$ da recomendação [ITU-T G.107 2015].

Tabela 8: Parâmetros de referência de atraso [ITU-T Y.1541 2011].

\begin{tabular}{|l|c|c|}
\hline Atrasos & Delay (ms) & Observação \\
\hline Formação do pacote & 20 & Dois tempos para o tamanho do frame \\
\hline D-Jitter Buffer, médio & 25 & Centro dos 50ms do buffer \\
\hline & 45 & \\
\hline
\end{tabular}

O cálculo do parâmetro T da equação (1.5) leva em consideração os valores de atraso apresentados na Tabela 8 , que são respectivamente: tempo de formação do pacote (TPacket), tempo do buffer Jitter (TBuffer), estando o valor T (TAtrasoLan) definido na seção 6.1 e obtido através do cálculo realizado na seção 5.2 deste experimento.

$$
\begin{gathered}
T=\text { TPacket }+ \text { TAtrasoLan }+ \text { TBuffer } \\
T=20 m s+\text { TAtrasoLan }+25 m s
\end{gathered}
$$

É importante salientar que o valor TAtrasoLan da equação (1.5) é o tempo de propagação do pacote na rede, medido neste experimento a partir do momento em que o pacote é enviado pelo transmissor até o momento em que ele é percebido no receptor.

Com isto posto e aplicando-se os critérios da recomendação [ITU-T G.108 2008], foi realizado o cálculo do atraso fim-a-fim, determinando o fator $\mathrm{R}$ de uma chamada de voz a partir do modelo E do ITU-T.

\section{Resultados}

A Tabela 9 apresenta o resultado completo dos experimentos realizados. É possível observar que a variação na quantidade de fluxos VoIP simultâneos sobre o canal PLC gerou um aumento no atraso da rede, porém não causou impacto considerável na percepção do usuário da chamada VoIP, pois não excedeu ao limite determinado pela recomendação [ITU-T G.108 2008].

\begin{tabular}{|c|c|c|c|c|c|c|c|c|c|c|c|}
\hline & \multicolumn{11}{|c|}{ QUANTIDADE DE FLUXOS SIMULTÂNEOS } \\
\hline & 1 & 5 & 10 & 15 & 20 & 25 & 30 & 35 & 40 & 45 & 50 \\
\hline TAtrasolan (ms) & 4,23 & 4,56 & 5,12 & 5,53 & 5,97 & 6,41 & 6,86 & 7,30 & 7,75 & 8,19 & 8,63 \\
\hline TPacket (ms) & 20 & 20 & 20 & 20 & 20 & 20 & 20 & 20 & 20 & 20 & 20 \\
\hline TBuffer (ms) & 25 & 25 & 25 & 25 & 25 & 25 & 25 & 25 & 25 & 25 & 25 \\
\hline JTTER (ms) & 3,08 & 4,86 & 2,97 & 3,35 & 3,04 & 4,55 & 1,65 & 2,34 & 3,06 & 2,40 & 3,87 \\
\hline PERDAS $(p c t)$ & 0 & 0 & 0 & 0 & 0 & 0 & 0 & 0 & 1 & 0 & 3 \\
\hline FATOR R & 91,7 & 91,7 & 91,6 & 91,6 & 91,6 & 91,6 & 91,6 & 91,6 & 91,6 & 91,5 & 91,5 \\
\hline MOS & 4,39 & 4,39 & 4,38 & 4,38 & 4,38 & 4,38 & 4,38 & 4,38 & 4,38 & 4,37 & 4,37 \\
\hline
\end{tabular}

Tabela 9: Cálculo do Fator $\mathbf{R}$ e critério MOS equivalente [autoria própria].

Essa informação pode ser validada quando comparamos o valor do Fator $\mathrm{R}$ calculado na Tabela 9 com a satisfação do usuário apresentada na Tabela 10, retirada da recomendação [ITU-T G.107 2015]. 
Tabela 10: Satisfação do usuário segundo o Fator R [ITU-T G.107 2015].

\begin{tabular}{|c|c|}
\hline $\mathbf{R}$ & Satisfação do Usuário \\
\hline & Muito Satisfeito \\
\hline & Satisfeito \\
\hline & Alguns Usuários Insatisfeitos \\
\hline & Muitos Usuários Insatisfeitos \\
\hline & Quase Todos os Usuários Insatisfeitos \\
\hline & Não Recomendado \\
\hline
\end{tabular}

Conforme demonstra o Gráfico 1 a seguir, o atraso fim-a-fim oscilou em valores menores que $150 \mathrm{~ms}$, no Gráfico 2 o jitter sempre esteve abaixo de $5 \mathrm{~ms}$, portanto não houve impacto significativo na qualidade de transmissão das chamadas VoIP estabelecidas durante essa análise.

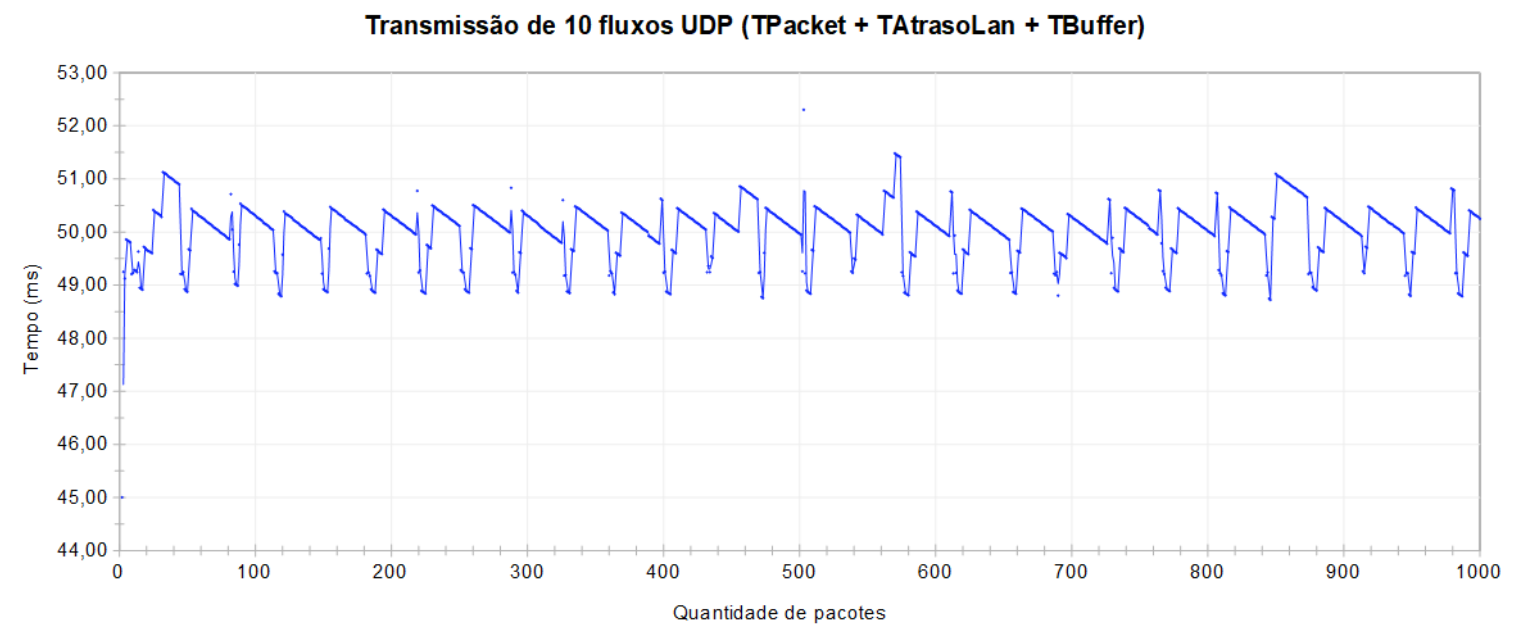

Gráfico 1: Transmissão de 10 fluxos UDP (Tpacket+TAtrasoLan+Tbuffer) [autoria própria]

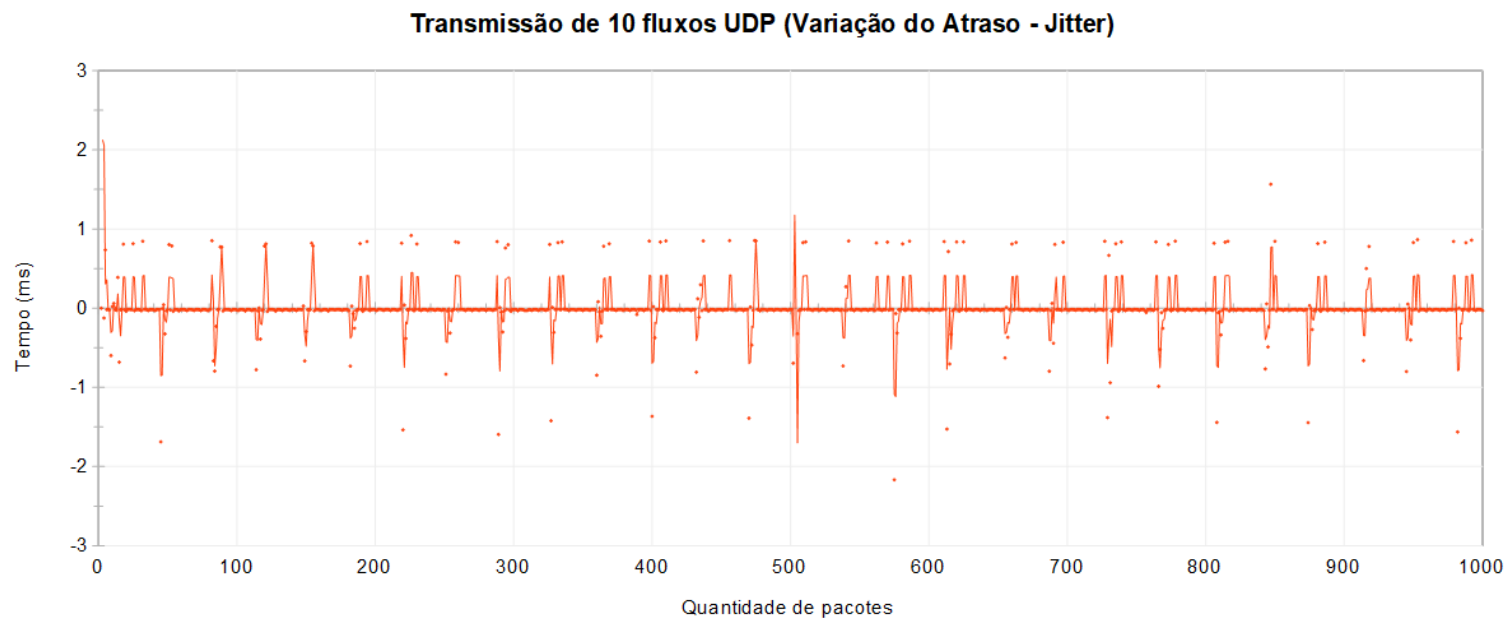

Gráfico 2: Transmissão de 10 fluxos UDP (Variação do Atraso - Jitter) [autoria própria]. 


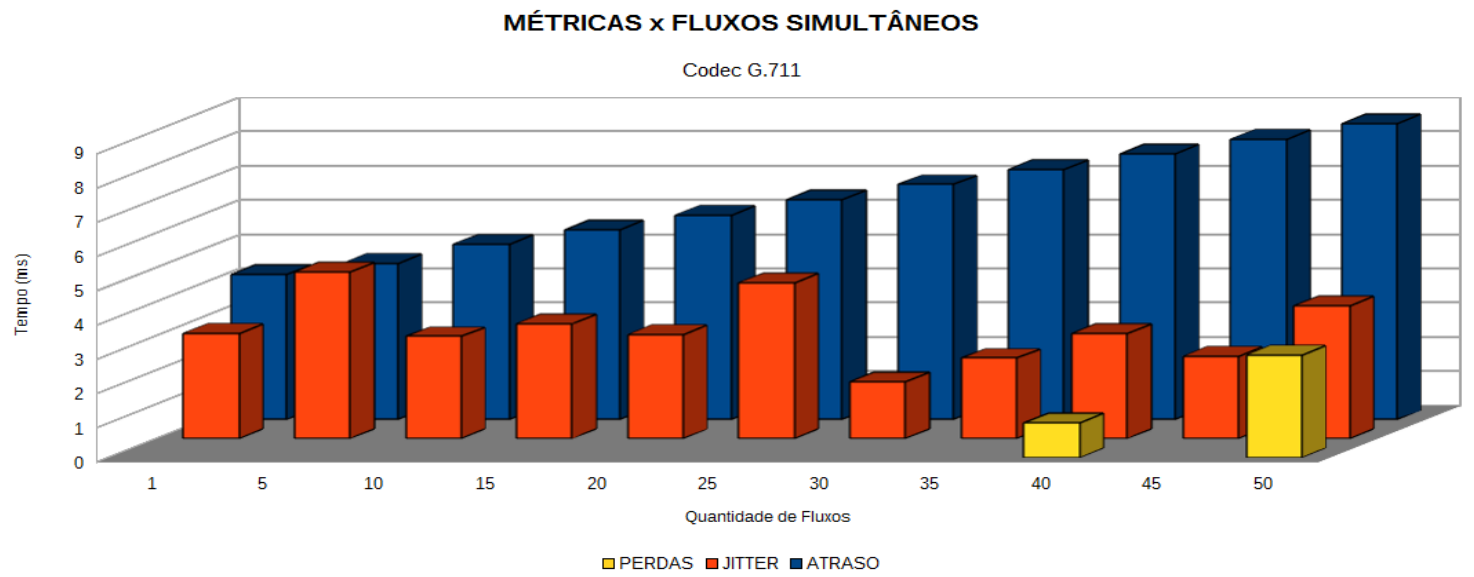

Gráfico 3: Métricas em função da quantidade de fluxos simultâneos [autoria própria].

O Gráfico 3 apresenta o resultado obtido com o aumento da quantidade de fluxos, onde percebesse o aumento do atraso fim-a-fim com o acréscimo das transmissões concorrentes, bem como o jitter oscilando dentro dos valores aceitáveis pelo ITU-T.

Por fim, com a variação dos fluxos concorrentes a partir de 40 transmissões simultâneas foi possível identificar perdas de pacotes, ainda assim não ocasionando um impacto significativo na qualidade das chamadas VoIP, pois este valor representa $0,001577 \%$ de perdas do total de pacotes transmitidos, abaixo do valor limite aceitável que é $0,5 \%$ [ITU-T Y.1541 2011].

\section{Conclusões}

Durante a realização desta pesquisa ficou evidenciado a importância da combinação das recomendações do ITU-T série G e Y para obtenção de uma análise de desempenho do fluxo de voz sobre redes de pacotes.

Contudo, conclui-se que o modelo $\mathrm{E}$ individualmente não é capaz de determinar o desempenho de uma chamada VoIP, portanto, é preciso utilizar as recomendações [ITUT G.108 2008], [ITU-T G.1020 2006], [ITU-T G.1050 2016] e [ITU-T G.114 2003], em conjunto com as recomendações [ITU-T Y.1540 2016] e [ITU-T Y.1541 2011] como peças complementares para análise de desempenho de voz sobre rede PLC.

Os resultados mostraram que as transmissões VoIP sobre o PLC possuem um bom desempenho para aplicações em ambiente residencial indoor.

Sendo assim, é possível concluir que para aplicações em tempo real no ambiente residencial a tecnologia PLC apresenta um desempenho satisfatório, pois o fator R da recomendação [ITU-T G.107 2015] indica que todas as chamadas obtiveram uma avaliação "Muito Satisfeito", tanto no fator R, quanto no critério MOS equivalente.

\section{Referências}

VIEIRA, Murillo. ALVES, Eliezer. (2015), Blindagem contra interferências eletromagnéticas provenientes do sistema de comunicação PLC. 2015 Pró-Reitoria de Pesquisa, Instituto Federal de Educação, Ciência e Tecnologia de Goiás. 
FIGUEIREDO Junior, PAULO de Tarcio. (2015), Expansão de rede local utilizando Power Line Communications (PLC). Universidade Federal do Ceará, Campus de Quixadá, Curso de Tecnologia em Redes de Computadores, CE.

ZATTAR, Haroldo. (2011), Análise, Medidas e Avaliações de desempenho de uma rede PLC em ambiente indoor de casas populares. 2011. 239 f. Tese (Doutorado em Engenharia Elétrica) - Programa de Pós-Graduação em Engenharia Elétrica, Universidade Federal de Uberlândia, Minas Gerais.

BRAGA, Marcelo Castellan. (2010), Análise de Jitter em tráfego VoIP utilizando o Modelo E, Universidade Federal Do Estado Do Rio De Janeiro Centro De Ciências Exatas E Tecnológicas Programa De Pós-Graduação Em Informática, RJ.

CAMARGO, Alexandre Lu. (2010), Avaliação da Tecnologia de Internet Sob Rede elétrica. 2010, 14 f. Programa de Pós-Graduação Em Redes e Segurança de Sistemas. Pontifícia Universidade Católica do Paraná, Curitiba.

ITU-T G.107 Recommendation (2015), Transmission Systems And Media, Digital Systems And Networks International telephone connections and circuits - Transmission planning and the E-model.

ITU-T G.1020 Recommendation (2006), Performance parameter definitions for quality of speech and other voiceband applications utilizing IP networks.

ITU-T G.108 Recommendation (2004), Application of the E-model: A planning guide. and Recommendation ITU-T G.108, Amendment 2: Appendix II - Planning examples regarding delay in packet-based networks.

ITU-T G.1050 Recommendation (2016), Network model for evaluating multimedia transmission performance over Internet Protocol.

ITU-T G.114 Recommendation (2003), One-way transmission time.

ITU-T Y.1540 Recommendation (2016), Network contribution to transaction time.

ITU-T Y.1541 Recommendation (2011), Network performance objectives for IP-based services.

ITU-T P.800 Recommendation (1996), Mean Opinion Score (MOS).

ITU-T P.830 Recommendation (1996), Subjective Performance Assessment of Telephone-Band and Wideband Digital Codecs.

ITU-T G.711 Recommendation Appendix I (1999), A high quality low-complexity algorithm for packet loss concealment with G.711.

RFC 3393 - Demichelis, C. and P. Chimento (2002), "IP Packet Delay / Variation Metric for IP Performance Metrics / (IPPM)"

WIRESHARK, Network Protocol Analyzer (2016) - http://wireshark.org,

JPERF, Check Network Performace - https://sourceforge.net/projects/jperf/, consulta em 07/06/16 